\title{
Adaptive Interference Cancel Filter for Evoked Potential Using High-Order Cumulants
}

\author{
Bor-Shyh Lin ${ }^{1}$, Bor-Shing Lin ${ }^{1}$, Fok-Ching Chong ${ }^{1}$, Feipei Lai ${ }^{1}$ \\ ${ }^{1}$ Institute of Electrical Engineering, National Taiwan University, Taipei, Taiwan
}

\begin{abstract}
This paper is to present evoked potential (EP) processing using adaptive interference cancel (AIC) filter with second and high order cumulants. In conventional ensemble averaging method, people have to conduct repetitively experiments to record the required data. Recently, the use of AIC structure with second statistics in processing EP has proved more efficiency than traditional averaging method, but it is sensitive to both of the reference signal statistics and the choice of step size. Thus, we proposed higher order statisticsbased AIC method to improve these disadvantages. This study was experimented in somatosensory EP corrupted with EEG. Gradient type algorithm is used in AIC method. Comparisons with AIC filter on second, third, fourth order statistics are also presented in this paper. We observed that AIC filter with third order statistics has better convergent performance for EP processing and is not sensitive to the selection of step size and reference input.
\end{abstract}

Keywords - evoked potential, adaptive interference cancel filter, high order cumulants

\section{INTRODUCTION}

Knowledge of Evoked potential of a patient is important in clinical electroencephalogram. When any of the visual, audio, and somatosensory nerve is stimulated, this external stimulus will conduct through the nervous system and reaches the cortex. This will then evoked the brain cell reflective electrical activities. However EP is a week signal and is always embedded in electroencephalogram (EEG). Thus, these reflexive stimuli are evoked from stimulation especially to visual, audio, and skin surface. Ensemble averaging (EA) is often used as the traditional method of processing EP. However, in recording, it needs many stimulus repetitions. It is also highly sensitive to minor changes in EP. To overcome these setbacks, recording evoked potential is substantially improved through the use of adaptive interference cancel filter [1]. A conventional AIC filter [2], which is denoted in this paper as AIC-NLMS algorithm, uses the normalized least mean squares algorithm and second order statistics. In practice, AIC-NLMS is problem-dependent. Its performance is sensitive to both of the reference signal statistics and the choice of step size. AIC-NLMS does not always converge faster than its transversal counterpart. Therefore, an AIC algorithm which is independent of both of the reference signal statistics and the choice of step size is important. Thus, adaptive interference cancel filter with high order statistics (AICHOS) [3] [4] is suggested to solve this problem due to their ability to preserve information of non-gaussian stationary random processes [3]. In poly-spectrum, gaussian signal components will be suppressed or eliminated, leaving nongaussian components substantially visible. Moreover, its auto-correlation function will also suppress phase information and AIC-HOS is not so sensitive to step size changes. In this study, we apply AIC-HOS with third and fourth order statistics, named AIC-TOS and AIC-FOS, to EP processing and compare their performance with AICNLMS.

\section{Methodology}

As shown in Fig.1, $\mathrm{x}(\mathrm{k})$ and $\mathrm{z}(\mathrm{k})$ denote primary and reference input in the block diagram structure of AIC-HOS, satisfying

$$
\begin{aligned}
& x(k)=s(k)+I(k)+n_{p}(k) \\
& z(k)=w(k)+n_{r}(k)
\end{aligned}
$$

Where $\mathrm{s}(\mathrm{k})$ denotes signal of interest, $\mathrm{I}(\mathrm{k})$ is non-gaussian interference, and $\mathrm{w}(\mathrm{k})$ is non-gaussian process signal. $\mathrm{n}_{\mathrm{p}}(\mathrm{k})$ and $\mathrm{n}_{\mathrm{r}}(\mathrm{k})$ are mean measurement noise and they are stationary, zero-mean, white or colored process.

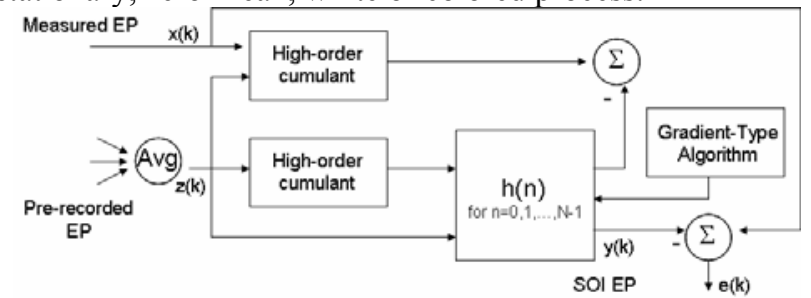

Fig. 1. The block diagram of AIC-HOS structure

And let $\mathrm{y}(\mathrm{k})$ be the N-tap adaptive filter output and $\mathbf{H}_{\mathbf{f}}=\{\mathrm{h}(\mathrm{n})\}$ for $n=0,1, \ldots, N-1$, is the adaptive filter coefficients. In practice, measured EP signal is used as primary input $\mathrm{x}(\mathrm{k})$ and the different average of pre-recorded EP signal is used as reference signal $\mathrm{z}(\mathrm{k})$. Here, we assume the relationship between interference and reference signal is a LTI transformation.

To develop the AIC-HOS algorithm, we assume there exists at least one order $n(n>2)$ such that the $n$th order cumulant [3] does not equal zero. Under this assumption, we design AICFOS. First, we find the fourth order joint cumulants of primary input and reference signal, $\mathrm{C}_{\mathrm{xzzz}}(\mathrm{m} 1, \mathrm{~m} 2, \mathrm{~m} 3)$ and $\mathrm{C}_{\mathrm{zzzz}}(\mathrm{m} 1, \mathrm{~m} 2, \mathrm{~m} 3)$. Then the cumulant of adaptive filter output can be written as

$$
C_{y z z z}(m 1, m 2, m 3)=\sum_{j=0}^{N-1} h(j) C_{z z z z}(j+m 1, j+m 2, j+m 3)
$$


The sum of the squared errors between two cumulants is used as the criterion of goodness, thus

$$
\xi_{g}=\sum_{m 1, m 2, m 3}\left[C_{x z z z}(m 1, m 2, m 3)-\sum_{j=0}^{N-1} h(j) C_{z z z z}(m 1, m 2, m 3)\right]^{2}
$$

We can rewrite (4) as matrix form

$$
\boldsymbol{\xi}=\left(\mathbf{C}_{x z z z}-\mathbf{C}_{z z z z} \mathbf{H}_{f}\right)^{T}\left(\mathbf{C}_{x z z z}-\mathbf{C}_{z z z z} \mathbf{H}_{f}\right)
$$

Then the gradient of the criterion and the weighted update equation is given by

$$
\begin{aligned}
\nabla_{f}(k)= & \frac{\partial \xi}{\partial \mathbf{H}_{f}(k)}=2\left(\mathbf{C}_{x z z z}^{T}-\mathbf{C}_{z z z z} \mathbf{H}_{f}(k)-\mathbf{C}_{z z z z}^{T} \mathbf{C}_{x z z z}\right) \\
& \mathbf{H}_{f}(k+1)=\mathbf{H}_{f}(k)-\mu \nabla_{f}(k)
\end{aligned}
$$

where $\mu$ is adaptive filter step size.

The algorithm of third order cumulant AIC filter is similar as AIC-FOS, but its criterion of goodness is given by

$$
\xi=\left(\mathbf{C}_{x z z}-\mathbf{C}_{z z z} \mathbf{H}_{f}\right)^{T}\left(\mathbf{C}_{x z z}-\mathbf{C}_{z z z} \mathbf{H}_{f}\right)
$$

But we have to take attention that the third order cumulants of reference signal are identically zero when the probability density function of the reference signal is symmetric [3].

\section{RESULTS}

We designate the average of a set of 4000 pre-recorded SEP data as the standard EP pattern. This pattern is mixed with different gain EEG signal to produce two sets of simulated input EP signal with SNR of $-30 \mathrm{db}$ and $-5 \mathrm{db}$. These two sets are used to represent VEP and AEP. We use the average of different amount simulated EP signal as the reference signal. The mean square error and signal-noiseratio results obtained after processing through AIC were checked with standard pattern.

\section{A. The performance between different adaptive filter step} size

In processing simulated EP via 8-taps and 16-taps AIC filter, we obtained the mean square error (MSE) results of AICNLMS, AIC-TOS, and AIC-FOS. These were the mean of 500 repeated experimental results obtained by changing the adaptive filter step size. Maximum likelihood estimation $\mathrm{SNR}_{\mathrm{ml}}$ [5] was used to evaluate converge performance.

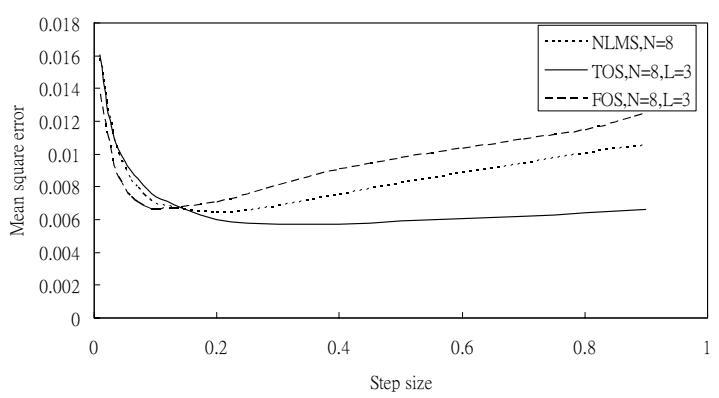

Fig. 2. The mean square error results by changing the step size via NLMS, TOS, FOS with filter taps $=8,-5 \mathrm{db}$ simulated $\mathrm{EP}$, and the data number of $\mathrm{EP}$ average used as reference signal is 1

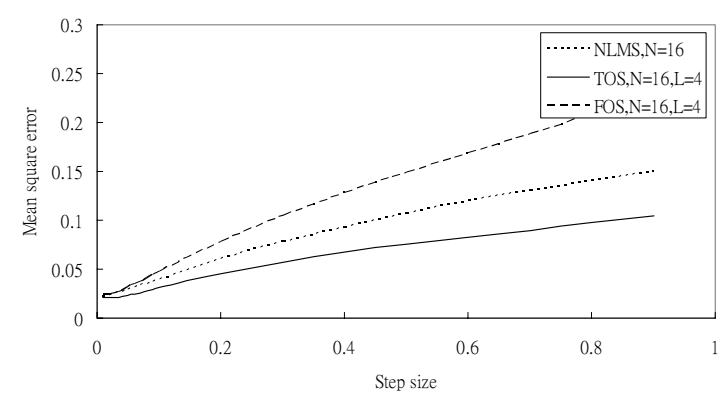

Fig. 3. The mean square error results by changing the step size via NLMS, TOS, FOS with filter taps $=16,-30 \mathrm{db}$ simulated EP, and the data number of EP average used as reference signal is 5

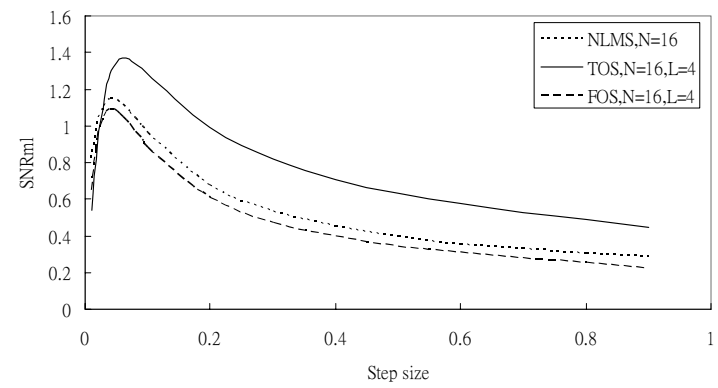

Fig. 4. The SNRml results by changing the step size via NLMS, TOS, FOS with filter taps $=16,-30 \mathrm{db}$ simulated $\mathrm{EP}$, and the data number of EP average used as reference signal is 5

\section{B. The performance between different reference signal}

In processing simulated EP via 16-taps AIC filter with step size $=0.05$, we obtained the mean square error (MSE) result by changing the data number of simulated EP average used as reference signal.

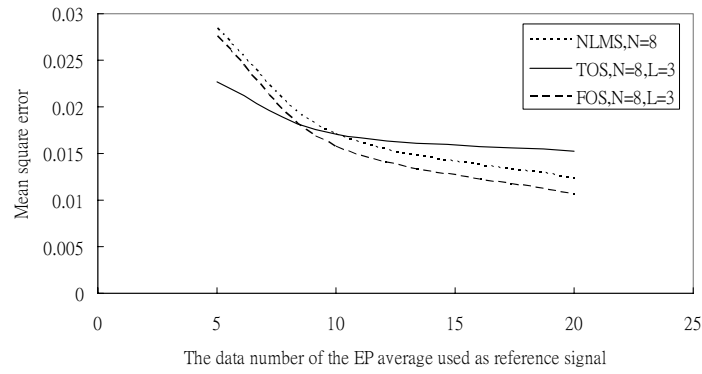

Fig. 5 (a). The mean square error results by changing the data number of reference signal via NLMS, TOS, FOS with step size $=0.05$, filter taps $=8$, and $-30 \mathrm{db}$ simulated EP 


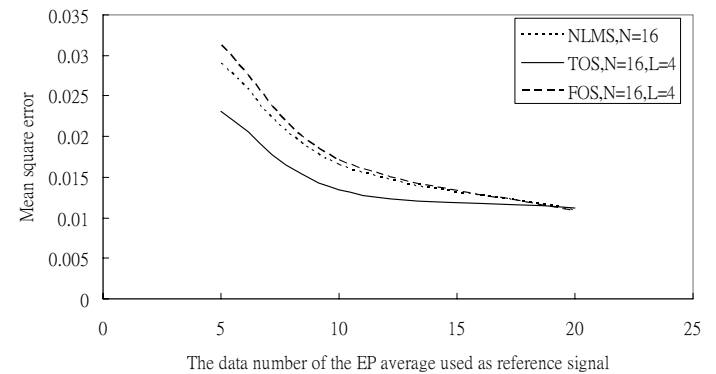

Fig. 5 (b). The mean square error results by changing the data number of reference signal via NLMS, TOS, FOS with step size $=0.05$, filter taps $=16$, and $-30 \mathrm{db}$ simulated EP

\section{DISCUSSION}

As shown in Fig.2 and Fig.3, AIC-TOS has better performance than AIC-NLMS and AIC-FOS and its MSE is non-sensitive when changing the filter step size. In Fig.4, it shows AIC-TOS has better convergence performance, and AIC-FOS convergence rate is slow especially in low SNR input signal.

Fig.5 is the comparison of MSE via AIC when changing the statistics of input signal. It clearly shows that the MSE of AIC-TOS is non-sensitive when the statistics of input signal changes. But AIC-NLMS and AIC-FOS seem to have better performance than AIC-TOS at step size $=0.05$ when the SNR of input signal is larger.

\section{CONCLUSION}

Finally, this result clearly showed that AIC-TOS is nonsensitive to the changing of filter step size and the statistics of input signal, although AIC-NLMS, AIC-TOS and AICFOS all have similar optimal performance. But in clinical usage, the stability of the EP processing via different choice of AIC parameters and input measured signal is more important. Thus AIC-TOS is realistic in clinical Electroencephalogy.

\section{REFERENCES}

[1] N V. Thakor, "Adaptive Filtering of Evoked Potentials", IEEE Trans. on Biomed. Eng.., vol.34 no.1, pp.6-12, 1987.

[2] Simon Haykin, Adaptive filter theory ( $3^{\text {rd }}$ edition). Englewood Cliffs, NJ: Prentice-Hall, 1996.

[3] C.L. Nikias and A. P. Petropulu, Higher-Order Spectra Analysis ( $3^{\text {rd }}$ edition). Englewood, NJ: Prentice-Hall, 1993.

[4] D. C. Shin, etc., "Adaptive Interference Canceller for Narrowband and Wideband Interferences Using Higher Order Statistics", IEEE Trans. on Signal Processing, Vol.42 ,No. 10, pp 2715-2728, October, 1994.

[5] R. Coppola, etc., "Signal to noise ratio and response variability measurements in single trial evoked potentials",
Electroencephlogr. Clin. Neurophysiol., Vol. 44, pp 214-218, 1978.. 